\title{
Cytology of Self Fusions in Hyphae of Phanerochaete velutina
}

\author{
By R. C. AYLMORE* AND N. K. TODD \\ Department of Biological Sciences, Washington Singer Laboratories, University of Exeter, \\ Perry Road, Exeter EX4 4QG, UK
}

(Received 30 August 1985; revised 1 December 1985)

\begin{abstract}
Hyphal fusions forming in secondary mycelia of Phanerochaete velutina were examined using combined light and electron microscopy. Only 'self' fusions, occurring between hyphal compartments derived from the same colony, were studied. At points of contact, fusions formed from a single opening which expanded radially by highly localized lysis of the surrounding cell wall. Fusion pore enlargement failed to reach the full hyphal diameter and a rim of undissolved cell wall remained marking the original point of contact. Within $2 \mathrm{~h}$ of this, the compartments became re-partitioned by septum formation, synthesis being associated with mitotic division and beginning precisely at the site of fusion pore expansion. Septa formed at fusions showed identical structure and development to those occurring in unfused compartments.
\end{abstract}

\section{INTRODUCTION}

Somatic incompatibility is a common phenomenon between genetically different mycelia of the higher fungi (reviewed by Lane, 1981; Rayner et al., 1984). We have concentrated on the cytology of this process in the wood-decaying basidiomycete Coriolus versicolor, which shows a marked incompatibility reaction in plate culture (Rayner \& Todd, 1977, 1979). Surprisingly, however, direct microscopical observation of fusing hyphae failed to reveal any differences in the cytological events which occurred between somatically compatible (self) and incompatible (non-self) anastomoses. Indeed, with the exception of a small proportion of those between homokaryons of complementary mating type, both self and non-self mycelial fusions exhibited the same highly regulated initial response, the so-called 'nuclear replacement reaction' (Aylmore, 1983; Aylmore \& Todd, 1984a). It appears, therefore, that in this species the somatic incompatibility reaction is either delayed, restricted to a few fusion segments only or occurs in cells not immediately involved in the fusion event. In view of this we have examined another basidiomycete, Phanerochaete velutina, a species in which the somatic incompatibility is known to be expressed directly, as lysis and cellular degeneration, at the site of hyphal anastomosis (Rayner et al., 1984).

Unlike $C$. versicolor, which exhibits both uni- and bi-nucleate compartments in the primary (monokaryotic) and secondary (dikaryotic) mycelial phases respectively, in $P$. velutina the hyphal compartments of both phases are multi-nucleate, i.e. the holocoenocytic condition (Boidin, 1971). Here, germinating basidiospores give rise to homokaryotic primary mycelia, mating-type compatible pairs of which interact to form morphologically distinct secondary mycelia. Anastomoses between different secondary mycelia, or between these and homokaryons, result in the somatic incompatibility reaction.

Ainsworth \& Rayner (1986) made a detailed study of living hyphae of $P$. velutina, using light microscopy. In this paper we examine the fine structure of self (compatible) fusions in secondary mycelia. These occur as part of normal development and therefore serve as 'controls' for those fusions (non-self) which result in a somatic incompatibility reaction, the details of which are given in the accompanying paper (Aylmore \& Todd, 1986). Previous ultrastructural work on this species has already revealed several interesting features, including unusually large microtubule bundles in vegetative hyphae (Aylmore et al., 1985). 


\section{METHODS}

Strains and culture conditions. Heterokaryotic secondary mycelia, synthesized from wild homokaryotic isolates of $P$. velutina, were supplied by A. M. Ainsworth, University of Bath. Stock mycelia were maintained at $25^{\circ} \mathrm{C}$, on $2.0 \%(\mathrm{w} / \mathrm{v})$ malt extract, solidified with $1.5 \%(\mathrm{w} / \mathrm{v})$ agar. For cytological work, mycelia were grown on cellophane membranes overlying $0.2 \%(\mathrm{w} / \mathrm{v})$ malt agar. Media and cellophane membranes were sterilized by autoclaving at $10 \mathrm{lbf} \mathrm{in}^{-2}(69 \mathrm{kPa})$ for $20 \mathrm{~min}$.

Light microscopy. A piece of cellophane $\left(10 \mathrm{~mm}^{2}\right)$ carrying a small area of mycelial monolayer was cut from the periphery of a single growing culture and loaded into a microculture chamber (for details see Aylmore \& Todd, $1984 b$ ). After overnight incubation at ambient temperature $\left(18-21^{\circ} \mathrm{C}\right.$ ), intramycelial ('self') fusions were located and followed using a Leitz Dialux microscope fitted with a Heine phase condenser. Specimens were examined with phase and bright-field optics. Photomicrographs were taken on Kodak Technical Pan 2415 film.

Electron microscopy. For combined light and electron microscopy, fused hyphae were followed to the appropriate stage under phase and bright-field optics before the whole mycelium was removed from the microculture chamber and processed for electron microscopy. After flat-embedding, the fusion was re-located and longitudinally sectioned. For details of these procedures see Aylmore \& Todd $(1984 b)$. Sections were stained with lead citrate (Reynolds, 1963) and examined at 60-80 kV with a JEM 100 S electron microscope.

\section{RESULTS}

The course of development of self fusions was followed. Our findings are based on at least 25 separate sets of observations.

\section{Light microscopy}

Hyphae of $P$. velutina formed fusions with high frequency: $85 \%$ were of the tip-to-tip type, involving two growing apices, one often forming in a lateral wall as a result of the telemorphotic action of the other (see Buller, 1933). In other instances, fusions were of the tip-to-side type, an extending apex fusing into a morphologically normal lateral wall, or appression types, an opening forming in regions of lateral wall contact with no apparent involvement of a recognizable growing point. Zygotropism (attracted growth of hyphae before contact) was frequently observed. The first visible opening between hyphae occurred within 20 min of initial contact and this enlarged during the following $15 \mathrm{~min}$ to leave a rim of cell wall material. In appression fusions, the hyphal walls could be in contact for several hours before fusion occurred. Cytoplasmic continuity, with exchange of nuclei, mitochondria and vacuoles through the opening, was evident for about $75 \mathrm{~min}$. However, within $90 \mathrm{~min}$ septum formation started, ingrowth beginning from the rim of undissolved wall marking the original site of the fusion pore. After a further $30 \mathrm{~min}$, septal swellings became evident and the cross-wall appeared to partition functionally the fused compartments (see Fig. $1 a-e$ ). Both hyphae remained viable, showing further growth and development with typical patterns of lateral branch formation and septation.

Exceptions to the behaviour outlined above were uncommon. In only one case did septa form in any position other than the precise site of the original fusion. Fusions occasionally remained open for extended periods, allowing free movement of organelles between compartments. In several instances septum synthesis failed, especially in compartments showing multiple fusions. It should be noted that, whilst many septa lacked clamp connections, a proportion possessed single, paired or whorled clamps. During development of these, septa formed only at the base of the hook and not at the point of fusion as described above. The septa synthesized at the points of hyphal fusion did not themselves form clamp cell structures.

It was not feasible to monitor accurately the behaviour of the numerous nuclei present in the fused compartments. Sometimes nuclei were seen to aggregate close to the site of anastomosis, although their origin and possible involvement in mitosis was not recorded. No evidence was obtained for the degeneration of specific nuclei as found in other species (Aylmore \& Todd, 1984a; Todd \& Aylmore, 1985).

\section{Electron microscopy}

Hyphae fixed at various stages of the fusion process are shown in Figs 1-3. A single opening formed in the region of wall contact, with complex regularly-spaced double-membrane systems 
enclosing amorphous material which were associated with the points of wall dissolution (arrowed in Figs $2 a$ and $2 b$ ). The fusion pores enlarged by radial expansion with erosion of the hyphal wall localized to the region immediately surrounding the growing opening; elaboration of the plasmalemma at these points was often evident (Figs $2 a$ and $2 b$ ). This resulted in full cytoplasmic continuity, nuclei and mitochondria traversing the fusion channel and microtubule bundles running between the two compartments (Fig. $2 e$ ). Pore enlargement failed to reach the full hyphal diameter and, at the end of this phase, a rim remained, derived from the coalescence of the original hyphal walls (Fig. $2 e-f$ ). Evidence for mitotic activity was rare. While electron microscopy confirmed that nuclei were often present close to the open pore channel, only one specimen showed a single division in this region (Fig. $2 f-g$ ).

The course of development of fusion-associated septa (see Fig. 3) is identical to that occurring in unfused growing compartments (see Fig. 4). Septal synthesis occurred centripetally, ingrowth always starting from the rim of residual wall material (Fig. $3 a-f$ ). Immature septal plates lacked a well-defined layered structure. Folds of double membrane, showing characteristic regular spacing, were associated with developing cross-walls (Figs $3 c$ and $4 a$ ) and the leading edge of the growing wall was often covered with amorphous electron-dense material (Fig. 4a-d). Mature septa showed typical dolipore structure with obvious parenthesomes and septal swellings, the majority in the more peripheral regions of the growing mycelia having unoccluded pore channels, whether formed at sites of fusion or in unfused hyphae (Figs $1 g$ and $4 f$ respectively). Septa formed at anastomoses could only be distinguished by the presence of a thickened outer rim (See Fig $1 g$ and $3 f$ ). Low-magnification electron micrographs of mature septa spanning points of fusion are shown in Figs $3(g)$ and $3(h)$. Longitudinal sections through single or double clamp connections confirmed that no cross-wall development occurred at points of hook cell fusion while those synthesized at the hook cell base possessed typical dolipore structure.

Ultrastructural examination failed to reveal evidence of the nucleus-specific degeneration which is associated with fusion in other species (Aylmore \& Todd, 1984a; Todd \& Aylmore, 1985). The fine structural details of these cells showed no atypical features and appeared identical to unfused, normally growing specimens.

\section{DISCUSSION}

The process of fusion in $P$. velutina involved the formation of a single opening which enlarged radially by highly localized dissolution of cell wall material. This establishes cytoplasmic continuity between the participating compartments, allowing free exchange of organelles. One notable feature of this species is the incomplete expansion of the fusion pore, leaving a rim of cell wall material which later acted as a site for septum synthesis.

How is the process of fusion initiated and controlled? Previous work on clamp cell fusion in Schizophyllum commune (Todd \& Aylmore, 1985), and unpublished observations in P. velutina, suggest an involvement of the apical vesicles. These accumulate close to the plasmalemma, at the point of contact, soon after the hyphae have touched but disappear shortly before the onset of wall dissolution. This rapid disappearance might suggest their coalescence with the plasmalemma and discharge of contents into the periplasmic space. Considering the accepted model of hyphal extension (Bartnicki-Garcia, 1973), it seems probable that the lytic enzymes involved in fusion are made available by this process. Indeed, this would be consistent with previous micrographs showing that vesicles can be found at points of lysis during various fusion events in fungi (e.g. Hawker \& Beckett, 1971; Harvey, 1975; Rijkenberg \& Truter, 1975; van der Valk \& Marchant, 1978).

Complex membrane-bound structures containing regions of amorphous electron-translucent material were usually associated with points of active cell wall dissolution (Figs. $2 a$ and $2 b$ ). These have been noted previously in fusion situations, apparently arising from plasmalemma invagination and elaboration (Aylmore, 1983; Aylmore \& Todd, 1984a). It is likely that they represent the sites where partially solubilized wall material is taken into the hyphae during the fusion process. It is of interest that structures with very similar appearances occur at sites of 

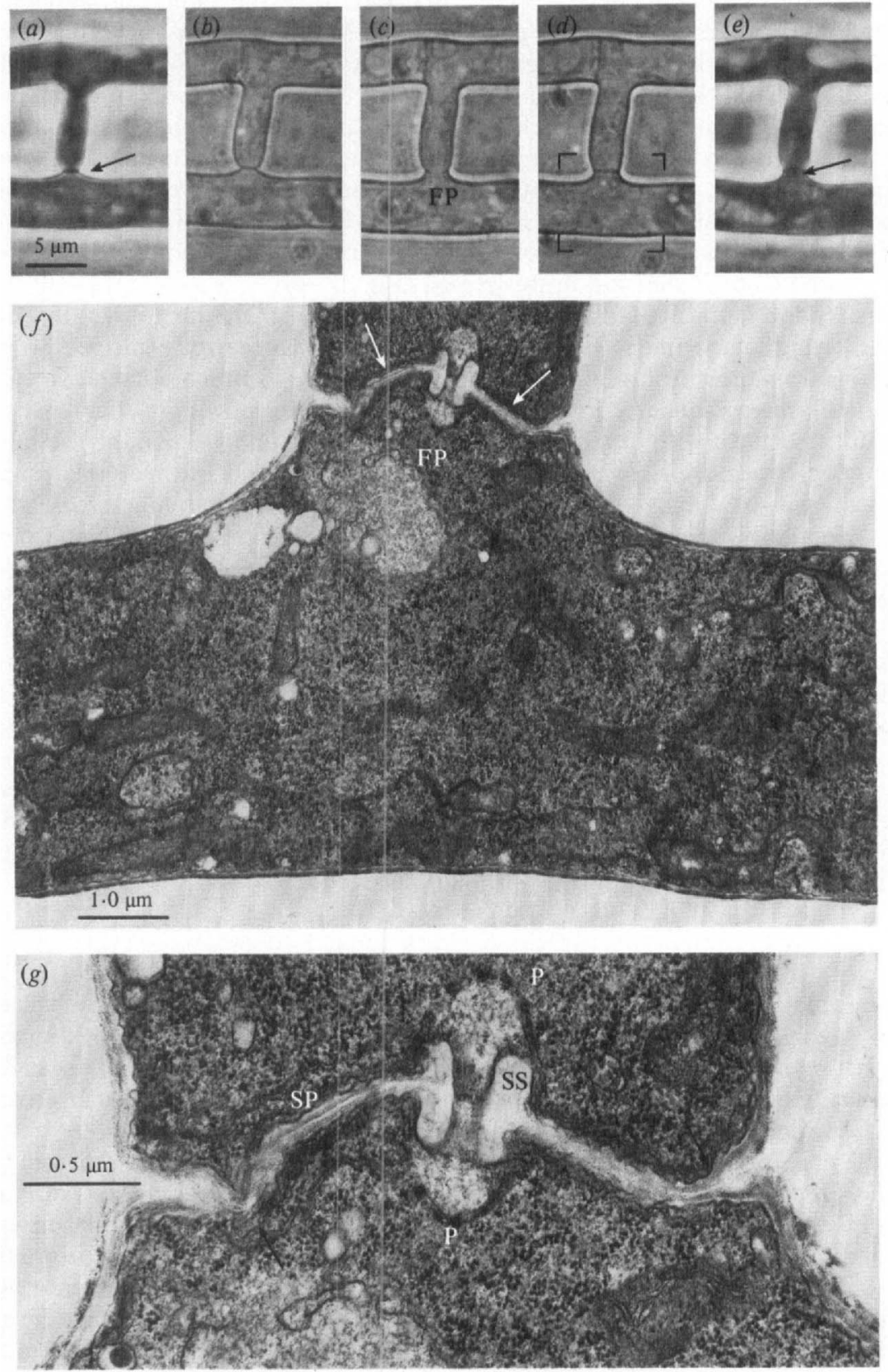

Fig. 1. Self fusions in $P$. velutina. ( $a-e)$ A sequence of photomicrographs taken, at the same magnification, with phase $(a, e)$ and bright-field $(b, c, d)$ optics. Initial contact between the extending apex and the nascent growing point [arrowed in $(a)$ ] resulted in wall appression $(b)$ and fusion $(c)$. Note incomplete expansion of the fusion pore (FP) after $30 \mathrm{~min}(c)$. Within $120 \mathrm{~min}$ of initial contact, septum formation at the site of fusion is evident $(d, e)$. The dolipore apparatus is arrowed $(e)$. $(f)$ Median section through the area boxed in $(d)$, specimen fixed $125 \mathrm{~min}$ after initial contact. Note the septum (arrowed) positioned across the fusion pore (FP). $(g)$ Micrograph, enlarged from $(f)$, showing typical dolipore apparatus with parenthesomes (P), septal swellings (SS) and open pore channel. The septal plate (SP) arises from the inner rim of the hyphal wall remaining from incomplete expansion of the fusion pore (arrowed). 

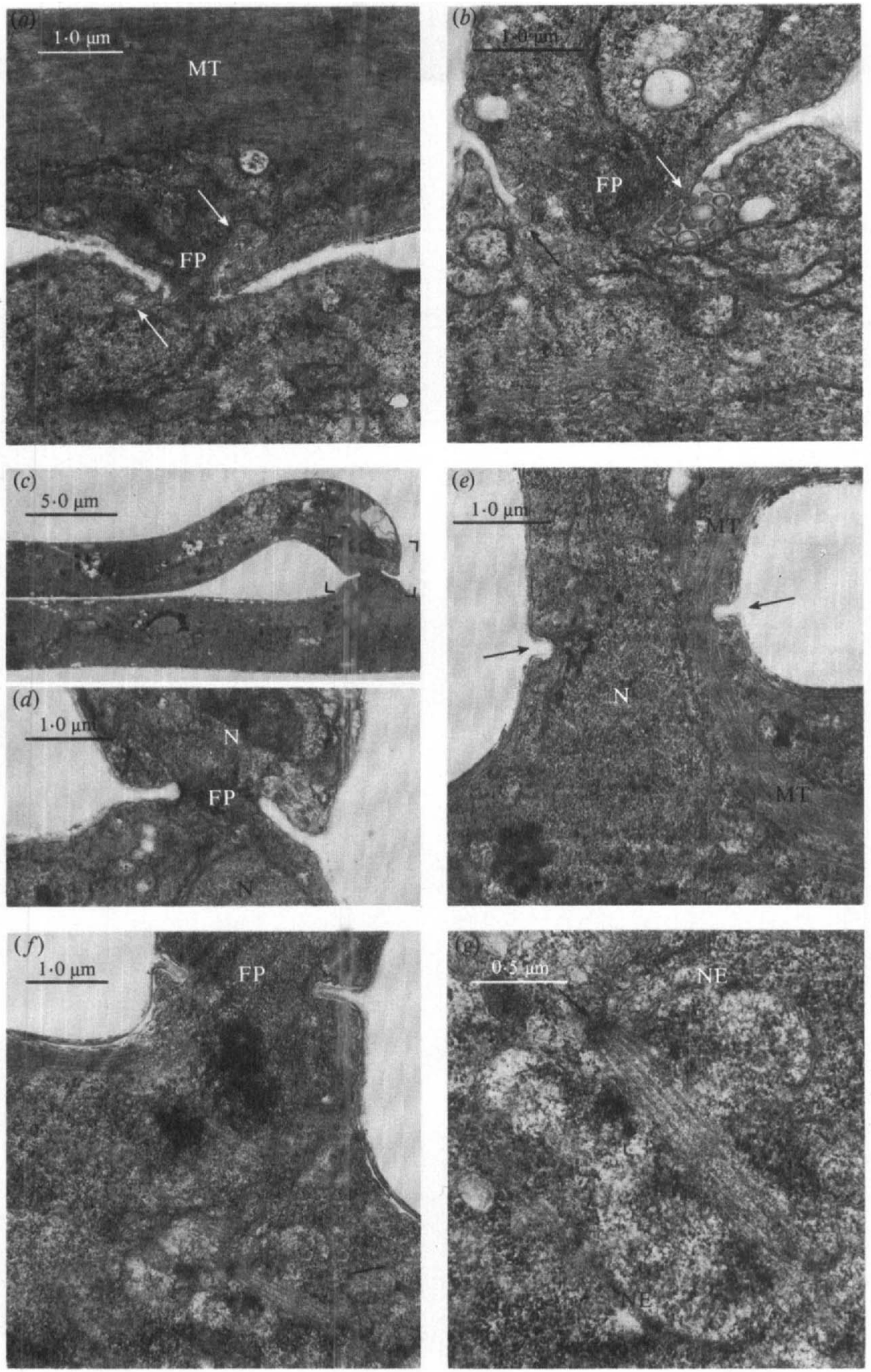

Fig. 2. Self fusions in P. velutina. (a) A single opening formed at the point of wall appression allowing cytoplasmic continuity. Note the presence of the amorphous membrane-associated material (arrowed) at the edge of the expanding fusion pore (FP), and the large microtubule bundle (MT). (b) Expanding fusion pore (FP) with invaginated plasmalemma marking points of wall lysis (arrowed). (c) Attracted growth before contact. $(d)$ The point of fusion enlarged from $(c)$. A nucleus $(n)$ occurred close to the fusion pore (FP). (e) Fully expanded fusion pore leaving a rim of undissolved wall material (arrowed). A nucleus $(\mathrm{N})$ and microtubule bundle (MT) traverse the opening. $(f)$ A mitotic nucleus (arrowed) close to a fully expanded fusion pore (FP). $(g)$ An enlargement from $(f)$ showing the dividing nucleus at meta/anaphase. Note the spindle arising from an electron-dense region (arrowed) associated with the intact nuclear envelope (NE). 

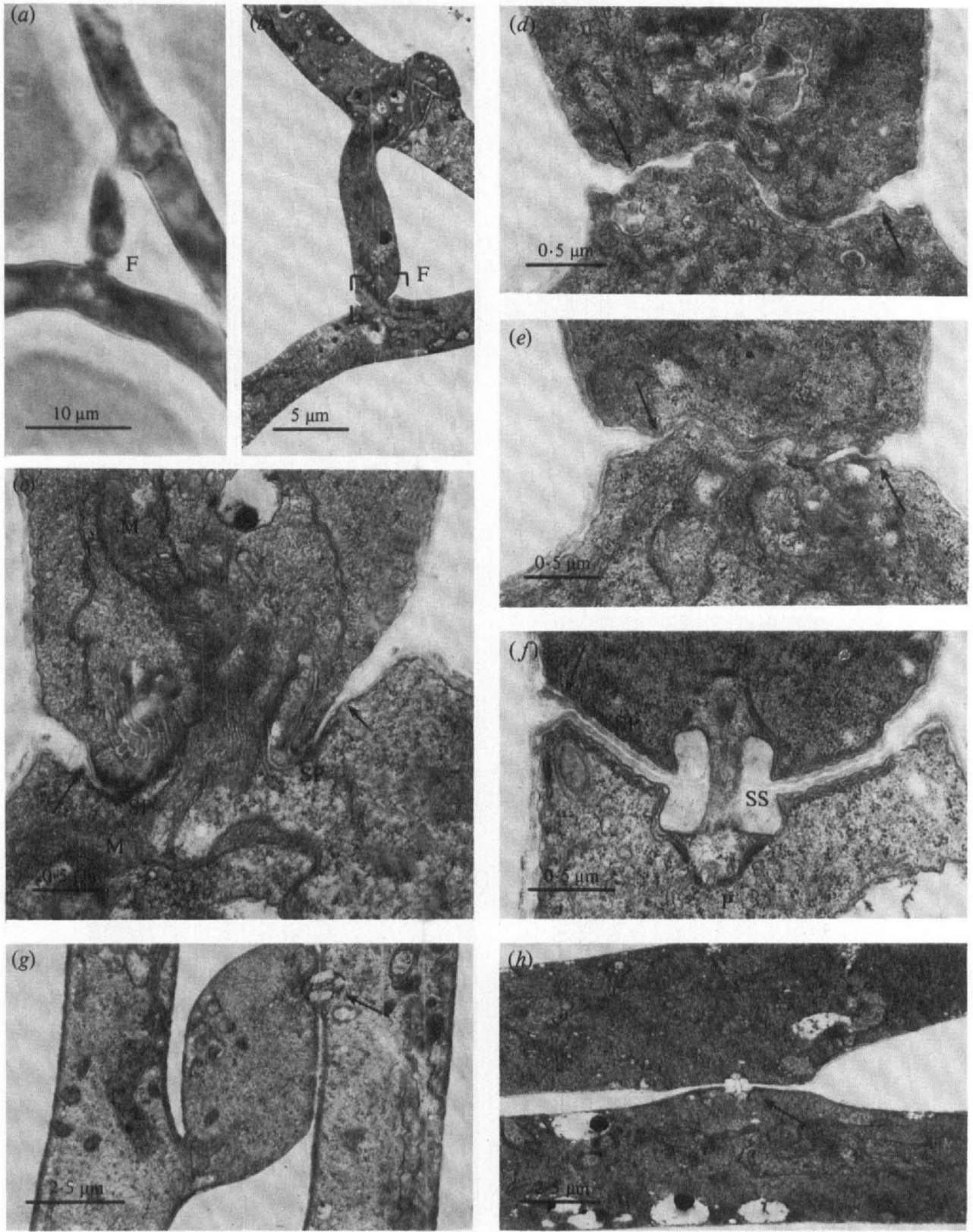

Fig. 3. Septum formation at sites of self hyphal fusions in $P$. velutina. $(a, b)$ Corresponding light and electron micrographs respectively of a tip-to-side fusion (F), fixed 93 min after contact. (c) Enlargement of bracketed area in $(b)$ showing septal ingrowth just beginning on the inner rim of the wall material remaining from fusion pore expansion (points arrowed). Note the presence of mitochondria (M) and the regions of amorphous material associated with membrane arising from the forming septal plate (SP). $(d, e)$ Near-median serial sections showing later stages of septum formation with a thin immature wall spanning the fusion (arrows show the sites of initiation). $(f)$ Mature dolipore septum formed across a fusion pore. Note the septal swellings (SS), open dolipore channel and parenthesomes (P). The outer electron-dense layers of the septal plate (SP) extend into the thickened region of hyphal wall remaining after fusion (arrowed). $(g, h)$ Dolipore septa (arrowed) formed across points of hyphal fusion. 

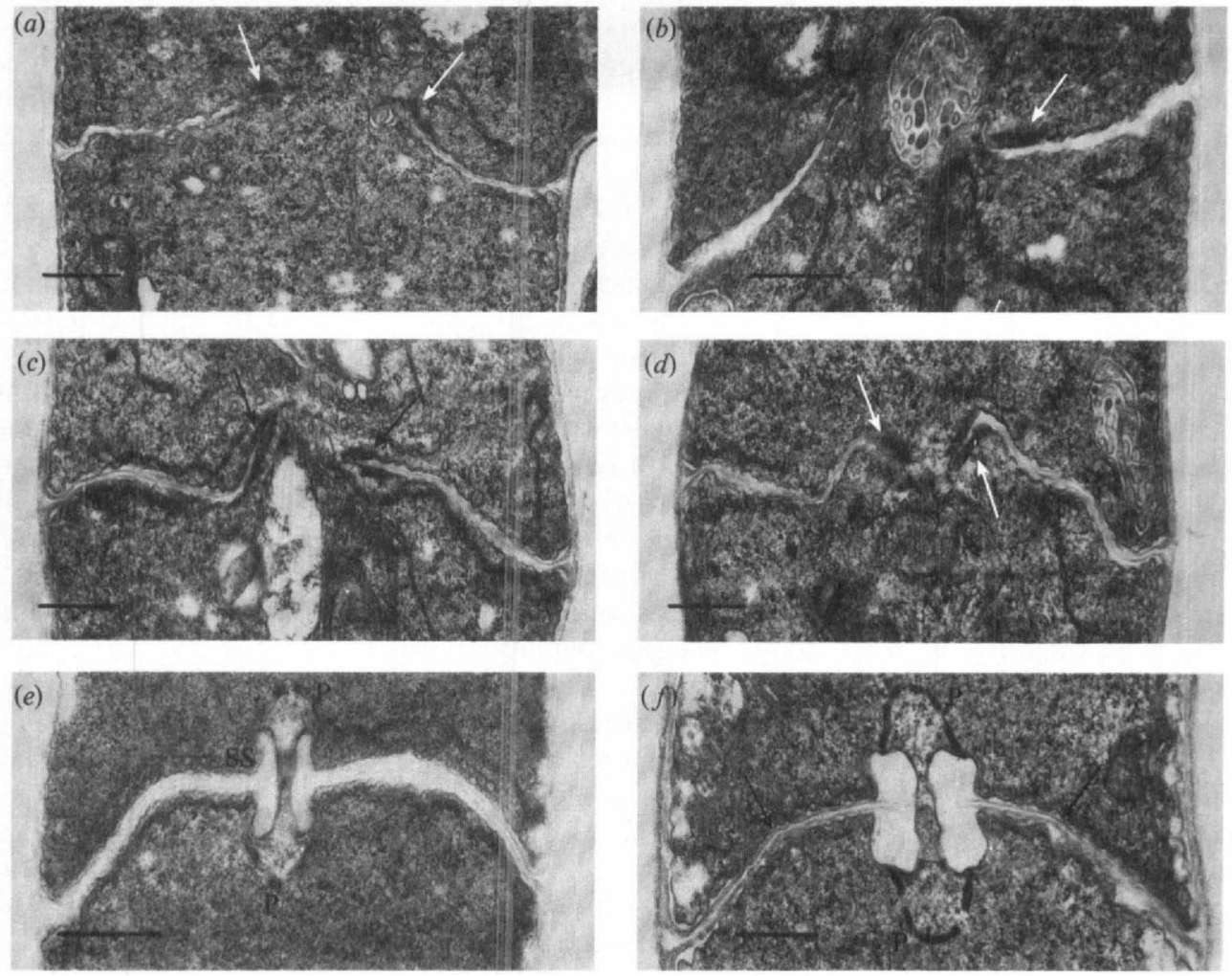

Fig. 4. Septum formation in unfused apical compartments of $P$. velutina. ( $a-d)$ Septa arise from the lateral walls by annular ingrowth. Note the amorphous electron-dense material (arrowed) confined to the inner regions of the developing walls. Large membrane-bound organelles occur close to points of wall deposition (see $b$ ). (e) Immature dolipore septum with partially developed septal swellings (SS) and intact parenthesomes (P). $(f)$ Mature dolipore septum. Note the unoccluded pore channel, obvious parenthesomes (P) and layered septal plate (arrowed). Bars, $0.5 \mu \mathrm{m}$.

septal synthesis (compare Figs $2 a$ and $4 b$ ), and are also found in association with mature walls and free in the cytoplasm. Although varied in fine structure, these may well represent a distinct class of organelles involved in general cell wall metabolism.

In $S$. commune and $C$. versicolor the 'nuclear replacement reaction' that follows fusion can be defined as a precise series of events (see Aylmore \& Todd, 1984a; Todd \& Aylmore, 1985). Similar behaviour is probably associated with fusion in Coprinus cinereus (Bensaude, 1918; cited in Papazian, 1958) and Typhula trifolii (Noble, 1937). However, the complexity of the multinucleate compartments of $P$. velutina makes a similarly detailed analysis of post-fusion behaviour difficult, but from this work several features were noted. Firstly, unlike our previous observations, we found no ultrastructural evidence for the degeneration of nuclei in either of the fused compartments. Secondly, as found previously, the act of fusion appears to serve as a mitogenic signal, with the division having a consistent spatial and temporal relationship to the fusion event. Figs $2(f)$ and $2(g)$ clearly confirm the association of a dividing nucleus with a point of anastomosis. This results in the partitioning of the fused compartments. In true dikaryons, septum formation is unquestionably coupled to the position of the dividing nuclei, whether the division occurs in an extending apical compartment, or in association with fusion sites. The way in which the nuclei determine patterns of septation has been elucidated by the elegant studies of Girbardt (1979) who has demonstrated the induction of cytokinesis at the meta/anaphase stage of division. Considering the multinucleate compartments of $P$. velutina, therefore, the question arises as to how the septum is induced to form so precisely on the point of 
anastomosis. Karyokinesis in this system appears to be less rigidly coupled to septation, with the position of the cross-wall forming after fusion apparently being regulated more by the properties of the cell wall at that point rather than by the position of the dividing nuclei. It may be that mitoses result in a more general septation stimulus and that it is the partial lysis of the existing cell wall at the receding edge of the fusion pore, by enhancing zymogen activation or exposing primer sites for synthase activity, that later causes synthesis to occur preferentially at this point. Indeed, clamp connection fusion fails to induce any of the reactions found in hyphal fusions in both bi- and multi-nucleate heterokaryons. Since the fusion events apparently share a common mechanism, the different response of the nuclei might be connected with the post-mitotic conditions which inevitably prevail after clamp cell development.

Why do the fused compartments of basidiomycetes undergo this re-partitioning? If fusions function principally to enhance multi-directional translocation of materials around the mycelium, more efficient in a network than a series of unconnected radial elements (Buller, 1933), then the presence of septa at these points might afford some level of regulation of this process. The septa associated with fusions and those occurring during normal hyphal extension showed identical development and structure, forming by a process of centripetal ingrowth in a manner similar to that of other basidiomycetes (Moore \& Marchant, 1972; Patton \& Marchant, 1978). The majority, at least in the younger regions of mycelium examined here, were unoccluded, allowing full humoral continuity between compartments. The formation of electron-dense pore plugs during dolipore synthesis reported elsewhere (Moore \& Marchant, 1972) was not evident in this work. These structures are known to be formed as a result of hyphal injury (Aylmore et al., 1984) and play a role in confining the incompatible reaction in non-self fusions (see Aylmore \& Todd, 1986). In true dikaryons, nuclear replacement and septum formation apparently serves to maintain the bi-nucleate arrangement, restoring nuclear numbers in compartments that have fused (Aylmore \& Todd, 1984a). The behaviour of the multi-nucleate heterokaryons suggests a similar requirement to regulate nuclear numbers in the same situation, surprisingly, since, compared to true dikaryons, nuclear numbers in these fungi vary considerably during normal growth anyway. Such speculation serves to highlight the lack of information regarding nucleo-cytoplasmic interaction, cell-cycle regulation and the cell concept in fungi generally.

Clearly, self fusions are not simply a matter of vegetative plasmogamy, rather they signify the start of a highly ordered sequence that culminates in the restoration of the original compartments which remain in contact through the dolipore channel. This constitutes part of the normal process of mycelial development, occurring between genetically identical hyphae derived from a single isolate. Such fusions cause no disruption to hyphal ultrastructure. By contrast, when the hyphae involved are not genetically similar, being derived from distinct secondary mycelia, an entirely different response occurs, resulting from the action of somatic incompatibility (Todd \& Rayner, 1980; Rayner et al., 1984). These events are described in the accompanying paper.

We wish to thank Drs A. D. M. Rayner and A. M. Ainsworth for their valuable discussions. This work was supported by the Science and Engineering Research Council.

\section{REFERENCES}

Ainsworth, A. M. \& RaYner, A. D. M. (1986) Responses of living hyphae associated with self and non-self fusions in the basidiomycete Phanerochaete velutina. Journal of General Microbiology 132, 191 201.

Aylmore, R. C. (1983). Hyphal fusion in Coriolus versicolor. PhD thesis, University of Exeter.

AylmoRe, R. C. \& TodD, N. K. (1984a). Hyphal fusion in Coriolus versicolor. In The Ecology and Physiology of the Fungal Mycelium. Symposium of the British Mycological Society, vol. 8, pp. 103-125.
Edited by D. H. Jennings \& A. D. M. Rayner. Cambridge: Cambridge University Press.

AYLMORE, R. C. \& TODD, N. K. (1984b). A microculture chamber and improved method for combined light and electron microscopy of filamentous fungi. Journal of Microbiological Methods 2, 317322.

AylmoRe, R. C. \& TODD, N. K. (1986). Cytology of non-self fusions and somatic incompatibility in Phanerochaete velutina. Journal of General Microbiology 132, 581-591. 
Aylmore, R. C., Wakley, G. E. \& Todd, N. K. (1984). Septal sealing in the basidiomycete Coriolus versicolor. Journal of General Microbiology 130, 29752982.

Aylmore, R. C., Ainsworth, A. M. \& Todd, N. K. (1985). Microtubule bundles in Phanerochaete velutina. Transactions of the British Mycological Society 84, 372-374.

BARTNICKI-GARCIA, S. (1973). Fundamental aspects of hyphal morphogenesis. Symposium of the Society for General Microbiology 23, 245-267.

BENSAUDE, M. (1918). Recherches sur le cycle écolutif et la sexualité chez les Basidiomycètes. Thèse Neumours.

BoIDIN, J. (1971). Nuclear behaviour in the mycelium and the evolution of the basidiomycetes. In Evolution in the Higher Basidiomycetes, pp. 129-148. Edited by R. H. Petersen. Knoxville: University of Tennessee Press.

Buller, A. H. R. (1933). Researches on Fungi, vol. 3. London: Longmans.

GIRBARDT, M. (1979). A microfilamentous septal belt (FSB) during induction of cytokinesis in Trametes versicolor (L. ex Fr.). Experimental Mycology 3, 215228.

HARVEY, I. C. (1975). Development and germination of chlamydospores in Pleiochaete setosa. Transactions of the British Mycological Society 64, 489-495.

Hawker, L. E. \& Beckett, A. (1971). Fine structure and development of the zygospore of Rhizopus sexualis (Smith) Callen. Philosophical Transactions of the Royal Society. Series B 263, 71-100.

LANE, E. B. (1981). Somatic incompatibility in fungi and myxomycetes. In The Fungal Nucleus. Symposium of the British Mycological Society, vol. 5, pp. 239-258. Edited by K. Gull \& S. G. Oliver. Cambridge: Cambridge University Press.

MOORE, R. T. \& MARChANT, R. (1972). Ultrastructural characterisation of the basidiomycete septum of Polyporus biennis. Canadian Journal of Botany $\mathbf{5 0}$. 2463-2469.

NoBle, M. (1937). The morphology and cytology of Typhula trifolii (Rost.). Annals of Botany 1, 67-98.
Papazian, H. P. (1958). The genetics of Basidiomycetes. Advances in Genetics 9, 41-69.

Patton, A. M. \& Marchant, R. (1978). An ultrastructural study of septal development in hyphae of Polyporus biennis. Archives of Microbiology 118, 271277.

RAYNER, A. D. M. \& TodD, N. K. (1977). Intraspecific antagonism in natural populations of wood-decaying Basidiomycetes. Journal of General Microbiology 103, 85-90.

Rayner, A. D. M. \& Todd, N. K. (1979). Population and community structure and dynamics of fungi in decaying wood. Advances in Botanical Research 7 , 334-420.

Rayner, A. D. M., Coates, D., Ainsworth, A. M., Adams, T. J. H., Williams, E. N. D. \& TodD, N. K. (1984). The biological consequences of the individualistic mycelium. In The Ecology and Physiology of the Fungal Mycelium. Symposium of the British Mycological Society, vol. 8, pp. 509-540. Edited by D. J. Jennings \& A. D. M. Rayner. Cambridge: Cambridge University Press.

REYNoldS, E. S. (1963). The use of lead citrate at high $\mathrm{pH}$ as an electron opaque stain in electron microscopy. Journal of Cell Biology 17, 208-213.

Rijkenberg, F. H. J. \& Truter, S. J. (1975). Cell fusion in the aecium of Puccinia sorghi. Protoplasma 83, 233 $\cdots 246$.

Todd, N. K. \& Aylmore, R. C. (1985). Cytology of hyphal interactions and reactions in Schizophyllum commune. In Developmental Biology of the Agarics. Symposium of the British Mycological Society, vol. 10, pp. 231-248. Edited by D. Moore \& L. A. Casselton. Cambridge: Cambridge University Press.

Todd, N. K. \& Rayner, A. D. M. (1980). Fungal individualism. Science Progress 66, 331-354.

VALK, P. VAN DER \& MARChant, R. (1978). Hyphal ultrastructure in the fruit body primordia of the basidiomycetes Schizophyllum commune and Coprinus cinereus. Protoplasma 95, 57-72. 